\title{
Optimisation of post-drawing treatments by means of neutron diffraction
}

\author{
J. Ruiz-Hervias ，J.M. Atienza , M. Elices , E.C. Oliver \\ Departamento de Ciencia de Materiales, UPM, E.T.S.I. Caminos, Canales y Puertos, \\ c/Profesor Aranguren s/n, E-28040 Madrid, Spain \\ ISIS Facility, CCLRC Rutherford Appleton Laboratory, Chilton, Didcot OXII OQX, United Kingdom
}

\begin{abstract}
The mechanical properties and the durability of cold-drawn eutectoid wires (especially in aggressive environments) are influenced by the residual stresses generated during the drawing process. Steelmakers have devised procedures (thermomechanical treatments after drawing) attempting to relieve them in order to improve wire performance. In this work neutron diffraction measurements have been used to ascertain the role of temperature and applied force - during post-drawing treatments - on the residual stresses of five rod batches with different treatments. The results show that conventional thermomechanical treatments are successful in relieving the residual stresses created by cold-drawing, although these procedures can be improved by changing the temperature or the stretching force. Knowledge of the residual stress profiles after these changes is a useful tool to improve the thermomechanical treatments instead of the empirical procedures used currently.
\end{abstract}

Keywords: Residual stresses; Neutron diffraction; X-ray diffraction (XRD); Eutectoid steels; Cold-drawing

\section{Introduction}

Eutectoid cold-drawn steels can be seen as - in present day terminology - nano-composite, nano-laminate materials, endowed with outstanding properties of strength and toughness that are still amazing to the modern metallurgist and materials engineer . Pearlitic cold-drawn wires and strands are the active tendons in prestressed concrete structures, support the tensile stresses in suspension and stayed bridges, and form the cables in mine shafts and off-shore petroleum production

Cold-drawn steel wires suffer a large plastic deformation during the drawing process. After drawing, strains tend to recover but if hampered somewhere by previous plastic deformation, a field of residual strains - and hence, stresses - may appear . The mechanical properties of cold-drawn eutectoid wires are controlled largely by the microstructure developed during processing and, to some extend, by the residual stresses generated in the drawing process. It is known that such stresses influence stress relaxation losses over time subcritical crack propagation in fatigue life, and environmentally assisted cracking

The role of these residual stresses is of such significance that steelmakers have devised procedures - still mainly heuristic to try to control them after cold-drawing. In the case of the prestressing industry, they attempt to modify the stress fields resulting from drawing by heating and/or stretching the wires

The purpose of this paper is to provide original data on the effect of different thermomechanical post-drawing treatments on the residual stresses. Such results are based on neutron diffraction experiments that have provided data until now unattainable.

\section{Experimental}

\subsection{Research programme}

The thermomechanical treatments applied by steelmakers to relieve residual stresses during cold-drawing are based on stretching and heating the wires during a short time period. This procedure - commercially secret - improves the mechanical properties and enhances the durability of steel wires in the presence of aggressive environments. 
To ascertain the role of temperature and tensile stress - during post-drawing treatments - on the residual stresses, five rod batches with different post-drawing thermomechanical treatments were produced for this research: the first one (called $S$, standard) was made by stretching the rods at 0.50 of the rupture load, at a temperature of $400^{\circ} \mathrm{C}$. The second and third batches were manufactured by stretching at different values, one at 0.38 of the rupture load (called LF, low force) and another one at 0.64 of the rupture load (called HF, high force), while keeping rod temperature at $400^{\circ} \mathrm{C}$. The fourth and fifth batches were processed at different temperatures while maintaining the stretching force at 0.50 of the rupture load: the fourth was heated at $330^{\circ} \mathrm{C}$ (called LT, low temperature) and the fifth heated up to $460^{\circ} \mathrm{C}$ (called $\mathrm{HT}$, high temperature). In addition, the "asdrawn" sample (called D) was also investigated. A summary of these thermomechanical treatments and its notation is given in Table 1.

\subsection{Material}

The samples were supplied by EMESA (Arteixo, La Coruña, Spain). The chemical composition of the steel used was 0.815 C, $0.231 \mathrm{Si}, 0.642 \mathrm{Mn}, 0.012 \mathrm{P}, 0.008 \mathrm{~S}, 0.044 \mathrm{~V}$ and $0.221 \mathrm{Cr}$ (mass\%). The initial rod (12 $\mathrm{mm}$ diameter) was subjected to six drawing passes to reach a final diameter of $7.0 \mathrm{~mm}$. The schedule of diameters (\% reduction in area) of the six drawing dies was: $11.6 \mathrm{~mm}(7 \%), 10.4 \mathrm{~mm}(20 \%), 9.3 \mathrm{~mm}(20 \%), 8.5 \mathrm{~mm} \mathrm{(18 \% ),}$
Table 1

Parameters of the thermomechanical heat treatments

\begin{tabular}{llll}
\hline Batch & Treatment & Force $\left(F_{\max }, \%\right)$ & Temperature $\left({ }^{\circ} \mathrm{C}\right)$ \\
\hline D & "as-drawn" & & \\
S & Standard treatment & 50 & 400 \\
LF & Low force & 38 & 400 \\
HF & High force & 64 & 400 \\
LT & Low temperature & 50 & 330 \\
HT & High temperature & 50 & 460 \\
\hline
\end{tabular}

$7.8 \mathrm{~mm}(16 \%)$ and $7.0 \mathrm{~mm}(20 \%)$. The drawing velocity was $44 \mathrm{~m} / \mathrm{s}$. Temperature was controlled during the process and the maximum value measured on the wire surface at the exit of the last die was $197^{\circ} \mathrm{C}$.

Longitudinal and transverse cross-sections were prepared for metallographic analysis. After polishing, samples were etched with Nital 2\%. Before SEM observation, a thin Au-Pd film was deposited on the surface of the samples by sputtering. In the micrographs obtained by SEM (Fig. 1) it can be seen that the rod microstructure is fully pearlitic, as corresponds to the eutectoid point $(0.8 \% \mathrm{C})$, with alternating nanosized ferrite $(\alpha-\mathrm{Fe})$ and cementite $\left(\mathrm{Fe}_{3} \mathrm{C}\right)$ lamellae (interlamellar spacing around $100 \mathrm{~nm}$ ). This is typical of cold-drawn eutectoid steel, the lamellae being aligned in the drawing direction, which coincides with the rod axis. The different post-drawing thermomechanical treatments do not produce visible changes in the microstructure. This is why only micrographs corresponding to
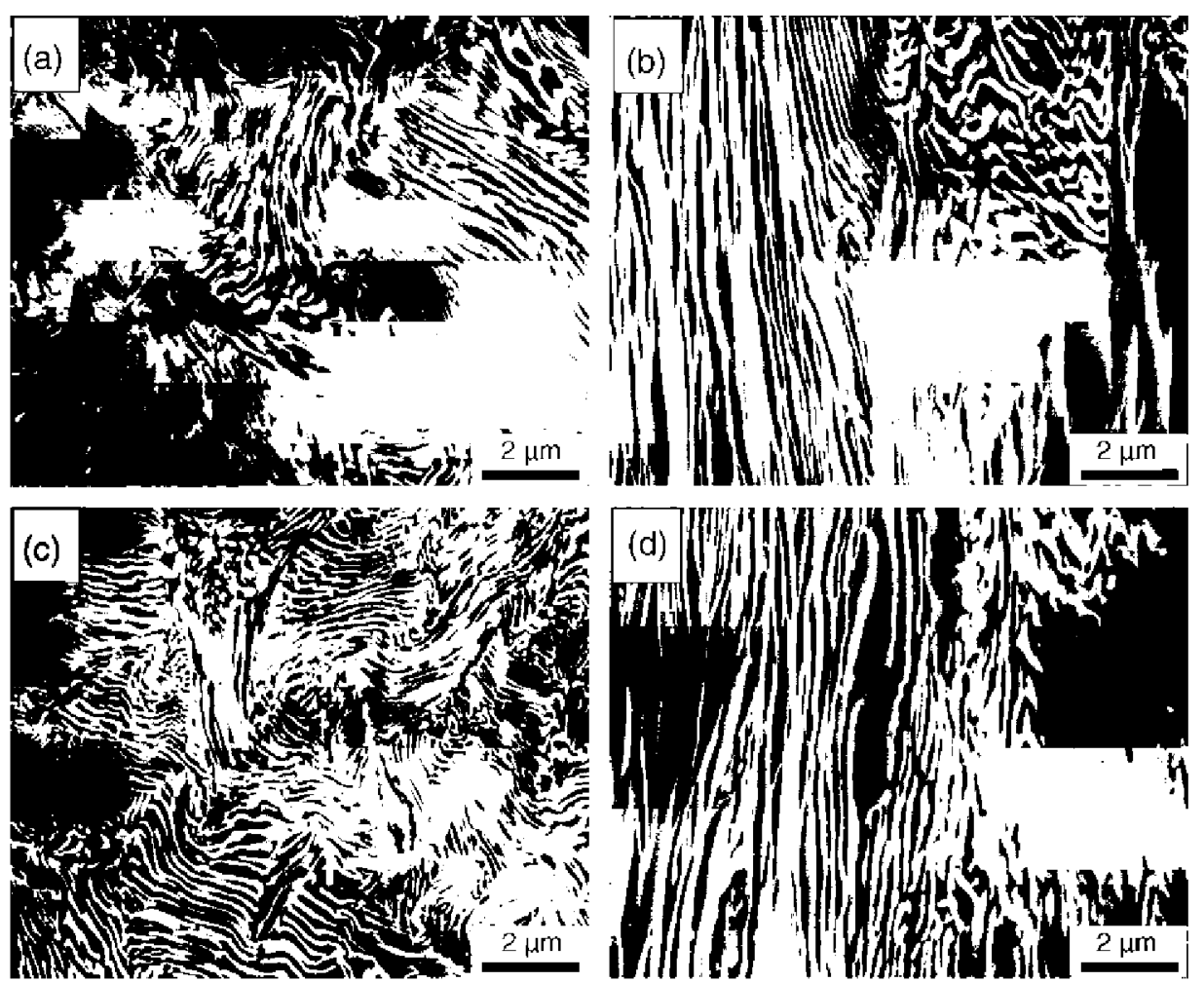

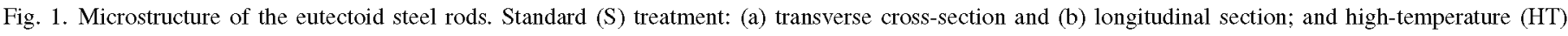

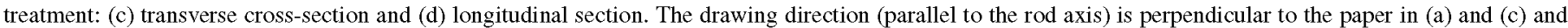

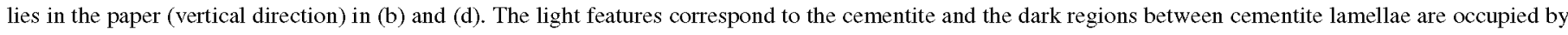
ferrite, which is etched by the reagent employed (Nital $2 \%$ ). 
Table 2

Mechanical properties of the different wires

\begin{tabular}{|c|c|c|c|c|c|}
\hline Batch & Diameter $(\mathrm{mm})$ & $\sigma_{\max }(\mathrm{MPa})$ & $\sigma_{0.2}(\mathrm{MPa})$ & $\varepsilon_{\max }(\%)$ & $\sigma_{0.2} / \sigma_{\max }(\%)$ \\
\hline $\mathrm{S}$ & 7.02 & 1817 & 1610 & 6.07 & 88.6 \\
\hline $\mathrm{LF}$ & 7.03 & 1814 & 1601 & 5.97 & 88.2 \\
\hline $\mathrm{HF}$ & 6.97 & 1820 & 1645 & 6.07 & 90.4 \\
\hline LT & 7.03 & 1833 & 1688 & 4.93 & 92.1 \\
\hline HT & 6.99 & 1784 & 1496 & 6.28 & 83.9 \\
\hline
\end{tabular}

the standard (S) and high-temperature (HT) samples are shown here.

Conventional tensile tests were performed with a universal testing machine to obtain the mechanical properties of the wires. The results (average of three tests) are given in Table 2 . As it is shown, the elastic limit $\sigma_{0.2}$ and the relation $\sigma_{0.2} / \sigma_{\max }$ are increased by reducing the temperature or increasing the applied force, while no significant changes are found in the tensile strength $\left(\sigma_{\max }\right)$. Increasing temperature improves the maximum uniform strain $\left(\varepsilon_{\max }\right)$ of the wires but it detrimentally affects the other properties.

\subsection{Residual stress measurement}

\subsubsection{X-ray diffraction}

Residual stresses were measured at the surface of the samples by X-ray diffraction. A Rigaku Strainflex diffractometer operated at $30 \mathrm{kV}$ and $8 \mathrm{~mA}$ was used. The $\alpha$-Fe reflection under study was (2 11 ), which produces a Bragg peak at $2 \theta=156.08^{\circ}$ for the $\mathrm{Cr} \mathrm{K} \alpha$ radiation employed $(\lambda=2.29 \mathrm{~A})$. Experiments were performed in $\Omega$-mode. Diffraction peaks corresponding to 10 values of $\psi$ angle ( $\sin ^{2} \psi$ interval from nearly $0-0.7$ ) were recorded for each residual stress measurement. Parallel beam optics and Soller slits $\left(1^{\circ}\right)$ were used both at the X-ray tube and the scintillation detector to minimize defocusing errors.

The diffractometer employed is a portable system. So, all the movements are performed by the goniometer, which includes the X-ray source and detector. A special experimental setup was designed for wires and rods, which consists of an $\mathrm{XY}$ table ( $\pm 0.01 \mathrm{~mm}$ precision) with two wire supports specially designed for turning the wire around its axis at $45^{\circ}$ steps. It allows one to perform measurements in large samples without having to cut them into small pieces. With this setup, residual stresses can be measured at several cross-sections along the same cylinder generatrix or in several generatrices corresponding to the same cross-section. In this way, the homogeneity of the surface stress state can be checked

\subsubsection{Neutron diffraction}

The neutron diffraction experiments presented in this paper were performed on the ENGIN-X Residual Stress Diffractometer, at the ISIS pulsed neutron and muon source (ISIS, CCLRC Rutherford Appleton Laboratory, Chilton, Didcot, UK). ENGIN-X is a $50 \mathrm{~m}$ flight path instrument. It sits on a curved "supermirror" neutron guide, with a large detector complement centered at $2 \theta=90^{\circ}$. It incorporates accurate and large capacity positioning equipment, and a range of sample environment equipment for engineering studies of materials. The neutron pulse is diffracted by the specimen and detected by two detector banks centered on horizontal scattering angles of $\pm 90^{\circ}$ relative to the incident beam, each with 1200 elements, made up of five vertically stacked units of 240 scintillator detectors. This allows the simultaneous collection of diffraction patterns (comprising several peaks) corresponding to two orthogonal sample directions for each measurement

Strain scanning was attempted in both phases of the rods, namely ferrite and cementite. However, cementite peaks could not be resolved in the patterns. Consequently, only ferrite peaks were indexed. Samples with a length to diameter ratio of 12-1 were cut from the rods. Measurements were performed in the central cross-section of the samples where the residual stresses
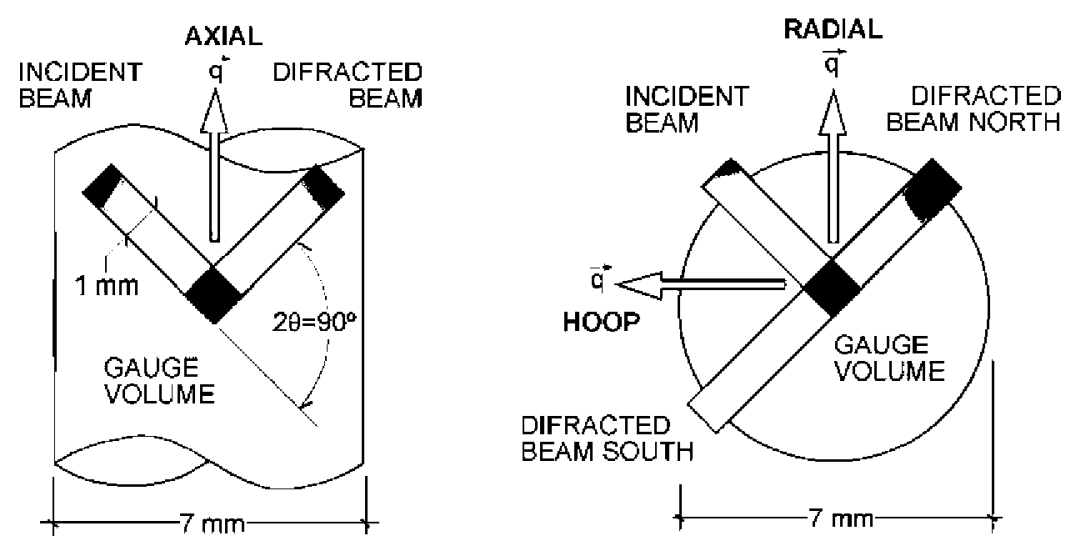

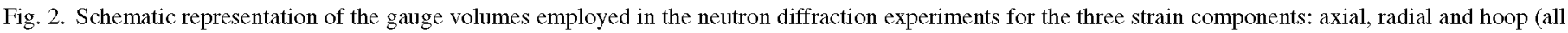
dimensions are scaled). There are two detectors, namely north and south detectors. 
(a) Radial/hoop orientation

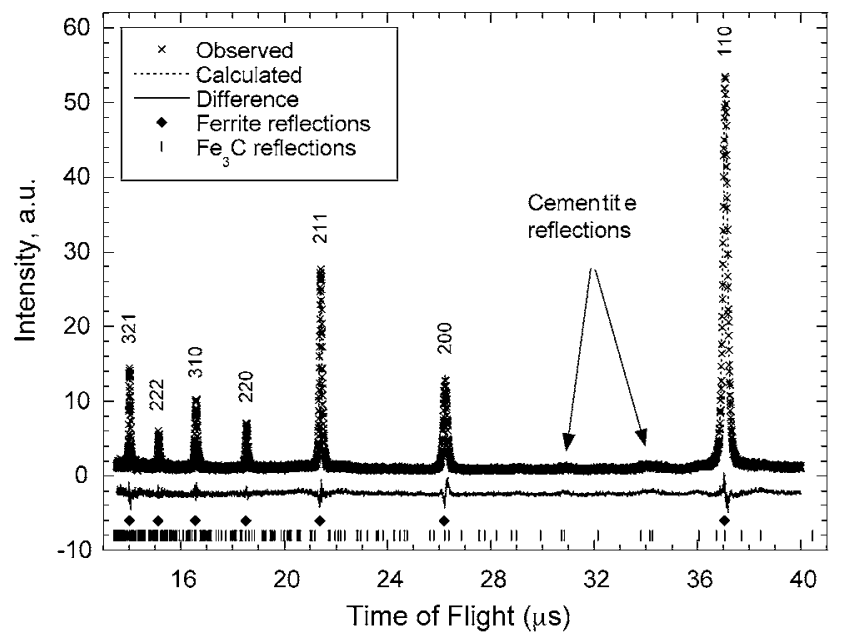

(b) Axial orientation

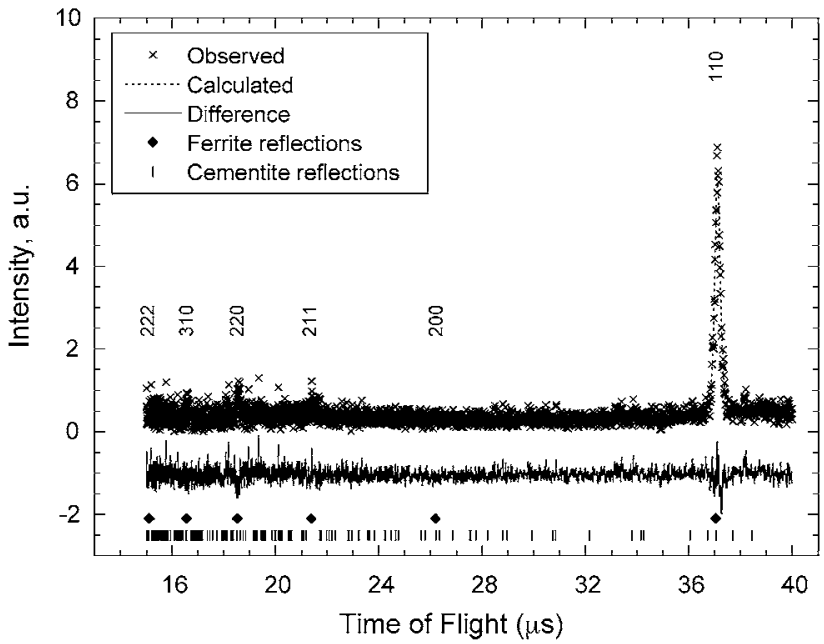

Fig. 3. Typical diffraction patterns recorded: (a) radial/hoop direction; (b) axial direction, including the fitting results and the ferrite and cementite reflections.

generated by cold-drawing were not affected by the cutting process . Ferrite lattice spacing was collected in the axial, radial and hoop directions of the samples, by measuring one point every $0.5 \mathrm{~mm}$. The strain scanning was carried out along the diameter perpendicular to the neutral plane of the rods, with $R=-3.5 \mathrm{~mm}$ corresponding to the inner radius and $R=3.5 \mathrm{~mm}$ being the outer radius. The gauge volume was defined by a combination of slits (incident beam) and collimators (diffracted beam). Different gauge volumes were employed for each direction (see Fig. 2), with the aim of achieving the same spatial resolution in all cases. In the axial measurements, a nominal gauge volume of $1 \mathrm{~mm} \times 1.5 \mathrm{~mm} \times 1 \mathrm{~mm}$ was used. For radial and hoop measurements, the beam could be enlarged vertically without loss in spatial resolution (due to the symmetry of the sample). So, in this case the slit dimensions were $1 \mathrm{~mm} \times 15 \mathrm{~mm}$ (incident) and $1 \mathrm{~mm}$ (receiving).

As explained above, the low intensity of the cementite peaks (hardly distinguishable from the background) prevented strain scanning in this phase. As an illustration, typical diffraction patterns in the radial/hoop and axial orientations are shown in Fig. 3. In the radial/hoop orientation (Fig. 3a), there is some evidence of very small cementite peaks between the $(200)$ and $(110)$ ferrite reflections. However, in the axial orientation (Fig. 3b), there is no sign of cementite, mainly because the intensity is much lower due to the smaller gauge volume employed. A strong (lll 10$)$ ferrite reflection is found in the axial orientation, as expected from texture development in cold-drawn steel wires ; the other reflections being almost negligible, as can be seen in Fig. 3 b. Rietveld refinement (with OpenGenie and GSAS software) was employed to analyze the results. In the radial and hoop orientations, at least seven ferrite peaks were fitted. Single peak analysis was performed in the axial direction, because only (1 10 ) reflection had enough intensity. The fitting is very good, as can be seen in the difference curve (observed minus calculated intensity) in Fig. $3 a$ and $b$.

Calibration was performed with a standard cerium oxide pow$\operatorname{der}(a=5.4114 \AA)$. The experimental lattice parameter obtained by Rietveld refinement provided two correction factors (one for each detector bank), which were applied to the measured values.

\section{Experimental results}

\subsection{Residual stresses in ferrite}

The strain for a given $(h k l)$ set of planes can be obtained from the variation in $d$-spacing:

$\varepsilon_{h k l}=\frac{d_{h k l}-d_{h k l}^{0}}{d_{h k l}^{0}}$

where $\varepsilon_{h k l}$ is the longitudinal strain in the direction of the scattering vector, and $d_{h k l}^{0}$ is the unstressed lattice spacing of the $h k l$ reflection. The stress is then computed through the appropriate elastic model, as will be explained below.

The unstressed lattice spacing, $d_{0}$, was computed from samples where the stresses had been relieved. To this end, several cuts were carried out by electro-discharge machining in samples from the $S$ and HT sets, respectively. The cuts left several isolated parallelepipeds $(2 \mathrm{~mm} \times 2 \mathrm{~mm} \times 15 \mathrm{~mm}$ ) where $d_{0}$ was measured in the radial/hoop orientation (gauge volume $1 \mathrm{~mm} \times 15 \mathrm{~mm} \times 1 \mathrm{~mm})$. The final $d_{0}$ employed in the calculations was an average of all the results corresponding to the different parallelepipeds in the samples employed. The resulting stress-free lattice parameter is $2.8665 \AA$, with a standard deviation of $0.0002 \AA$.

The residual strains originated by cold-drawing (D sample) are significantly reduced by the standard treatment ( $\mathrm{S}$ sample), as can be seen in Fig. 4. The strain profiles corresponding to the three directions become flatter and closer to zero, especially in the axial and hoop orientations. For example, the axial strain at the rod center changes from $-4 \times 10^{-3}$ in the D sample (Fig. 4a) to $-8 \times 10^{-4}$ in the $\mathrm{S}$ sample (Fig. $4 \mathrm{~b}$ ). 

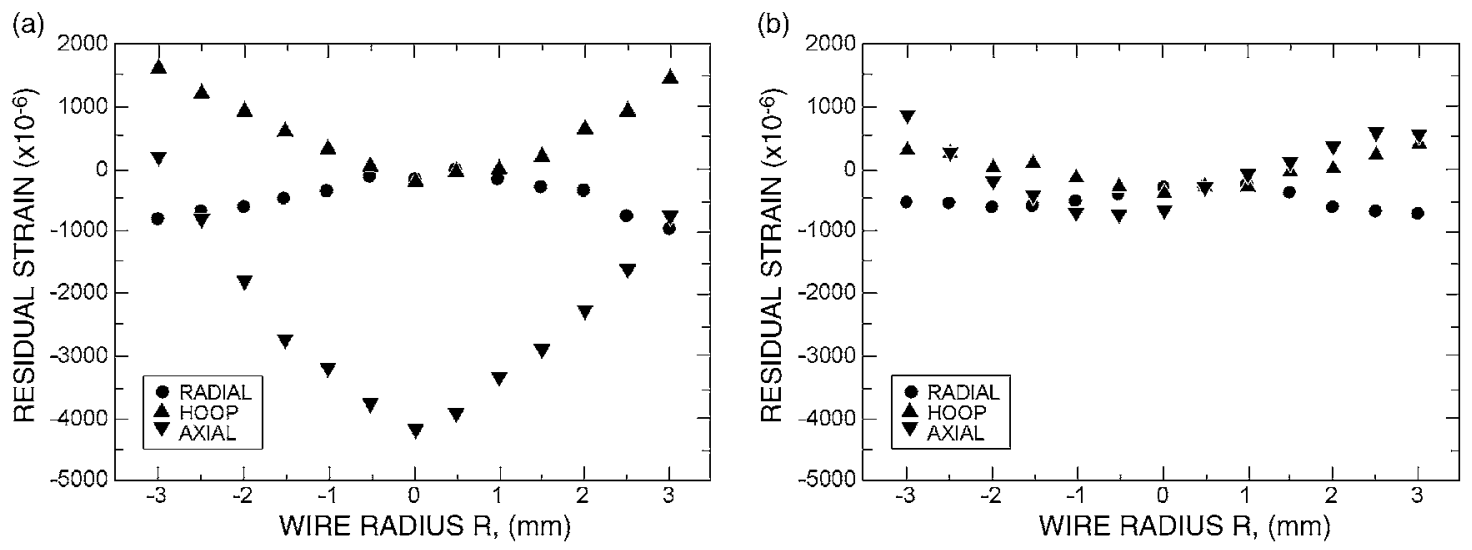

Fig. 4. Residual strains in the radial, hoop and axial direction as a function of the radial coordinate: (a) "as-drawn" (D) sample; (b) standard treatment (S) sample.

The variations to the standard thermomechanical treatment when the applied force is changed are shown in Fig. 5. If the applied force is reduced (LF sample), the strains are larger than the ones found in the standard treatment ( $\mathrm{S}$ sample), as shown in Fig. 5a. Conversely, if the applied force is increased (HF sample) the strain profiles are smoother and closer to zero than the ones found with the standard treatment ( $\mathrm{S}$ sample). As can be seen in Fig. 5b, the residual strains in the three directions are almost negligible in all points along the rod diameter.

If the temperature is changed and the applied force is kept constant, the resulting residual strains are depicted in Fig. 6. Similarly to the effect of varying the applied force, when temperature is decreased (LT sample, see Fig. 6a) the residual strains are larger than the ones measured with the standard treatment (S sample, see Fig. 4b). On the other hand, if the temperature is increased (HT sample), the strain profiles become almost flat. As can be seen in Fig. 6b, the radial and hoop strains in the high-temperature sample match the ones measured in the highforce sample, and the axial strain profile is displaced to positive values.

The residual stresses are calculated from the residual strains using linear elasticity . The elastic constants were computed from $S_{1}$ and $S_{2} / 2$ for the (110) ferrite reflection (single peak analysis for axial orientation measurements), which in turn were calculated from the single crystal elastic constants through the Kröner model , and their values are the following: $S_{1}=-1.2629$ and $S_{2} / 2=5.7155\left(\times 10^{-12} \mathrm{~Pa}^{-1}\right)$. For the radial and hoop data (obtained by Rietveld analysis), the elastic constants were calculated from the isotropic aggregate constants (Young's modulus and Poisson's ratio), based on a Eshelby-based approach for aggregates of arbitrary elastic symmetry [19], and their values are: $S_{1}=-1.3884$ and $S_{2} / 2=6.0920$ $\left(\times 10^{-12} \mathrm{~Pa}^{-1}\right)$.

The residual stresses in the ferrite phase are plotted in Figs. 7-9 as a function of the radial coordinate, $R$. As a result of cold-drawing, remarkable residual stress profiles are developed in the three directions, as can be seen in Fig. 7a, corresponding to the $\mathrm{D}$ sample. The steepest variations are found in the axial direction, the stresses varying from $-1200 \mathrm{MPa}$ at the rod center to approximately $100 \mathrm{MPa}$ at the surface. The hoop stresses are also compressive at the rod center (around $-600 \mathrm{MPa}$ ) and tensile at the surface (around $400 \mathrm{MPa}$ ). As expected from the strain results, the radial stress coincides with the hoop stress at the rod center, and reaches its maximum at the surface (almost zero in one side and around $-150 \mathrm{MPa}$ in the other). The standard treatment produces smoother stress profiles in the S sample (see Fig. 7b). The data corresponding to the three stress components are bound between -300 and $200 \mathrm{MPa}$, which are the minimum and maximum, respectively, of the axial stress. On the other hand, the hoop stress maximum is reduced to approxi-
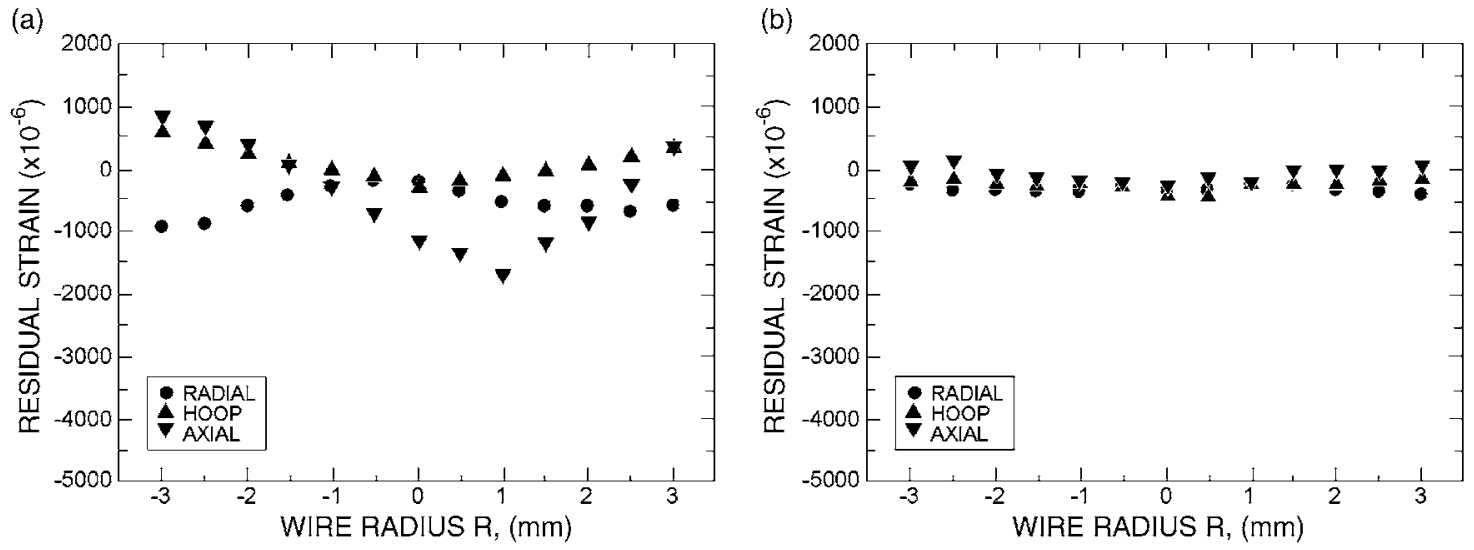

Fig. 5. Residual strains in the radial, hoop and axial direction as a function of the radial coordinate: (a) low-force (LF) sample; (b) high-force (HF) sample. 

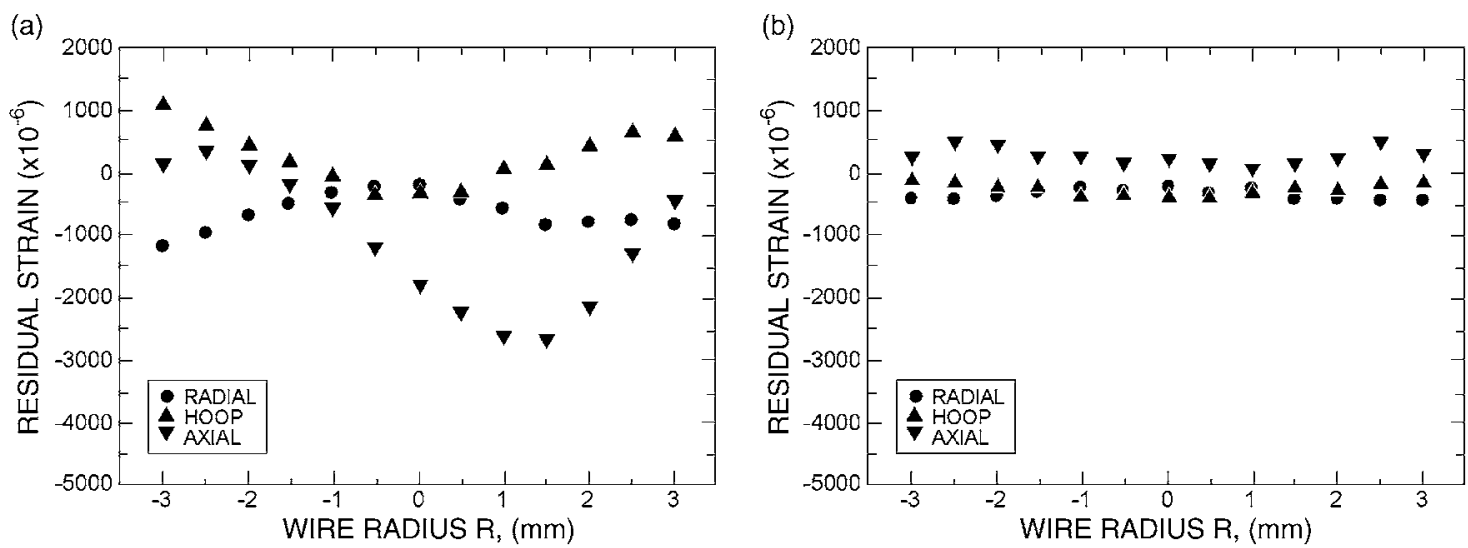

Fig. 6. Residual strains in the radial, hoop and axial direction as a function of the radial coordinate: (a) low-temperature (LT) sample; (b) high-temperature (HT) sample.
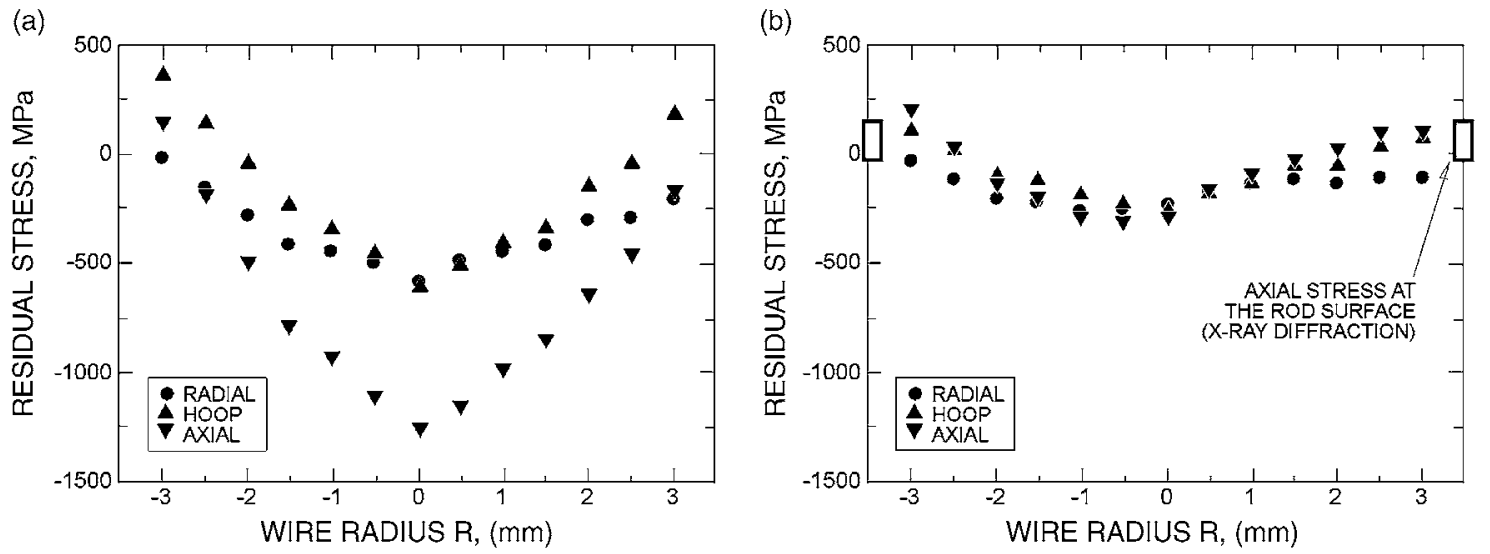

Fig. 7. Residual stresses in the radial, hoop and axial direction as a function of the radial coordinate: (a) "as-drawn" (D) sample; (b) standard treatment (S) sample. The axial stresses measured by $\mathrm{X}$-ray diffraction at the rod surface are also depicted.

mately $100 \mathrm{MPa}$ at the surface, and the minimum is $-250 \mathrm{MPa}$ at the rod center (same value as the radial stress in this location).

The consequences of changing the applied force while keeping constant the temperature of the thermomechanical treatment are shown in Fig. 8. If the applied force is reduced, the resulting post-drawing treatment (LF sample in Fig. 8a) produces steeper stress profiles than the standard one (S sample in Fig. 7b).
This is particularly noticeable in the axial component, where the minimum stress becomes lower than $-500 \mathrm{MPa}$ (compared to $-300 \mathrm{MPa}$ in the $\mathrm{S}$ sample). The radial and hoop stresses show small changes with respect to the values corresponding to the standard treatment. A clear profile asymmetry is found in the three directions. On the contrary, if the applied force is increased, the residual stresses are considerably reduced. The profiles for
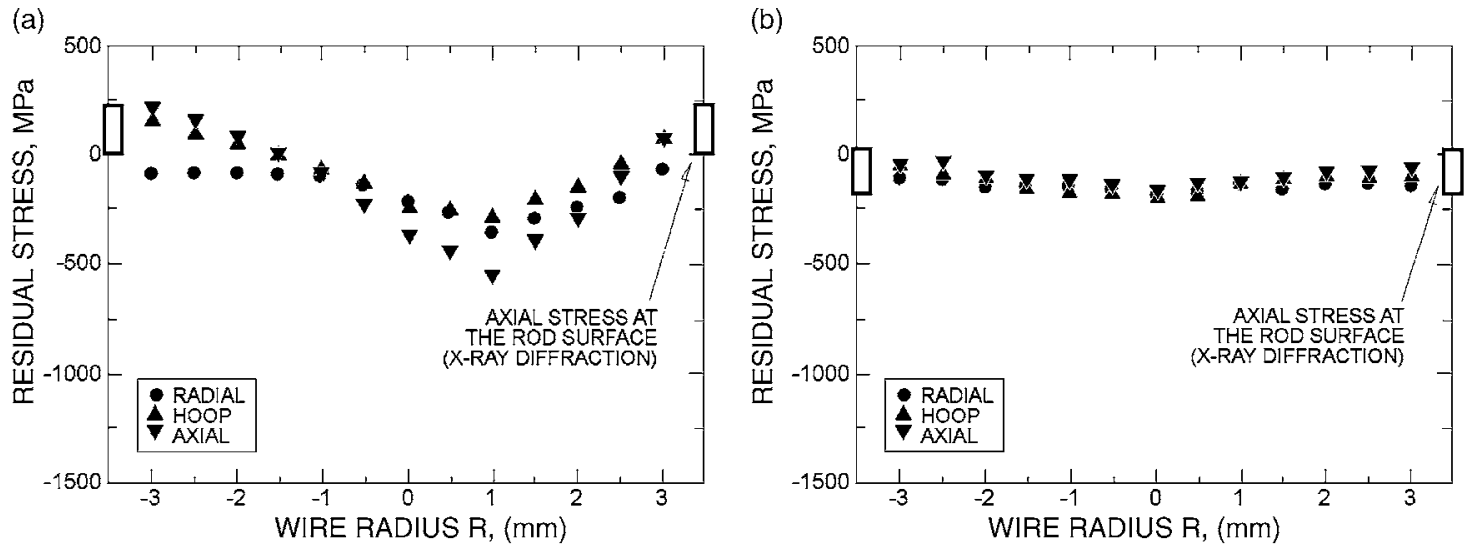

Fig. 8. Residual stresses in the radial, hoop and axial direction as a function of the radial coordinate: (a) low-force (LF) sample; (b) high-force (HF) sample. The axial stresses measured by $\mathrm{X}$-ray diffraction at the rod surface are also depicted. 
(a)

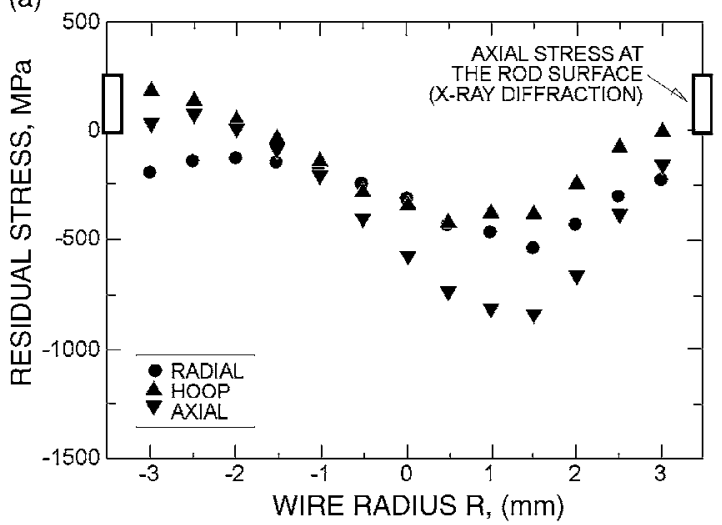

(b)

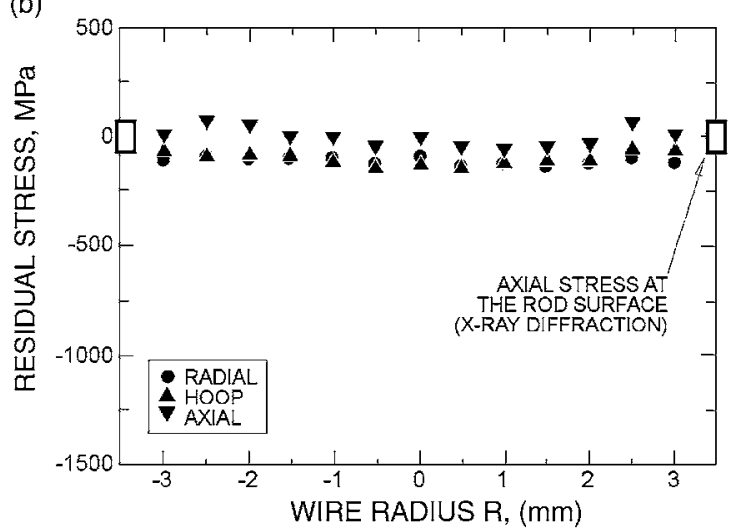

Fig. 9. Residual stresses in the radial, hoop and axial direction as a function of the radial coordinate: (a) low-temperature (LT) sample; (b) high-temperature (HT) sample. The axial stresses measured by X-ray diffraction at the rod surface are also depicted.

the three components are almost flat and their values bound in a narrow band of $200 \mathrm{MPa}$ width (from -200 to $0 \mathrm{MPa}$ ). The average stress (compressive for the three directions) is around $-100 \mathrm{MPa}$

If the temperature is changed and the applied force kept constant, the calculated residual stresses are the ones depicted in Fig. 9. In the low-temperature sample (Fig. 9a), the residual stresses are larger than in the standard treatment (Fig. 7b) for all stress components. The radial stress is always compressive with a minimum lower than $-500 \mathrm{MPa}$ displaced from the rod center, and a maximum around $-100 \mathrm{MPa}$ close to the surface. The hoop stress is tensile at the surface (around $200 \mathrm{MPa}$ ) and compressive at the center (approximately $-400 \mathrm{MPa}$ ). As happened with the low-force sample, the largest variations correspond to the axial component. The axial profile is noticeable asymmetric, with a minimum around $-900 \mathrm{MPa}$ close to the rod center, and a maximum around $100 \mathrm{MPa}$ at the surface. The results for the high-temperature sample are shown in Fig. 9b. In this case, the profiles are smoother than those of the standard sample (see Fig. 7b). Nevertheless, they are slightly different from the ones obtained for the high-force sample (see Fig. 8b). The radial and hoop stresses are very similar and rather constant, with an average value of $-100 \mathrm{MPa}$ (note that this does not violate stress balance because balancing stresses may exist in the cementite phase-see later). The axial stresses are slightly tensile, with values ranging from -50 to $100 \mathrm{MPa}$ approximately.

\section{Discussion}

The measured peak positions have a remarkable accuracy. The strain error is around $3 \times 10^{-5}$ for the radial and hoop components and $9 \times 10^{-5}$ for the axial one. Consequently, the error bars in the strain and stress results are very small (they have almost the same size as the symbols used in the plot). In addition, the axial residual stresses measured by X-ray diffraction at the rod surface agree with the neutron diffraction data, as can be seen in Figs. 7-9. This provides additional evidence of the reliability of the calculated residual stresses.

The small asymmetry observed in the residual stress profiles deserves some comments. Every sample was precisely posi- tioned $( \pm 0.1 \mathrm{~mm})$ by measuring entering curves through the rod surfaces by neutron diffraction. Moreover, it should be noted that there is excellent agreement between the radial and hoop strains exactly at the rod centre in all samples. Consequently, inaccuracies in sample positioning should be discarded. The most likely explanation of the asymmetry seems to rely on residual stresses associated with the shape of the sample - slightly bent due to cold-drawing. As it was explained above, the scanning diameter was perpendicular to the neutral plane of the $\operatorname{rod}(R=-3.5 \mathrm{~mm}$ is the inner radius and $R=3.5 \mathrm{~mm}$ the outer one). The post-drawing thermomechanical treatments not only attempt to reduce residual stresses but also to straighten the rods. The fact that after "soft" thermomechanical treatments (such as LF and LT) the rods still show some curvature and also exhibit the largest asymmetry in the residual stress profiles, supports this explanation.

Residual stresses in the direction of the rod axis - axial stresses - are meaningful because they add to the external loads during rod performance. Consequently, in the following discussion only axial stresses will be considered. Fig. 10 shows the profiles of axial stresses for the ferrite phase after cold-drawing (D). It can be noticed that, axial stresses can reach values as high as $-1200 \mathrm{MPa}$ at the rod centre and at the surface the stress changes sign and achieves tensile values. The authors have shown that this residual stress profile has some deleterious effects on the mechanical behaviour of the wires [3,9], as well as on their durability [13].

The profile of residual stresses after cold-drawing is improved by the standard (S) treatment (temperature around $400^{\circ} \mathrm{C}$ and stretching force around 0.50 of the rupture load), as shown in Fig. 10. The stress profile is flattened; stresses at the rod centre are close to $-300 \mathrm{MPa}$, although stresses at the surface are not substantially reduced. This type of profile improves the mechanical properties , the stress relaxation behaviour (a significant aspect for prestressing steels), and their susceptibility to environmental assisted cracking . As already mentioned, detailed values of the thermomechanical treatment used by steelmakers - temperature and stretching force-are largely empirical and not easily available in the open literature. Any additional information of the effect of these parameters on the final profile 


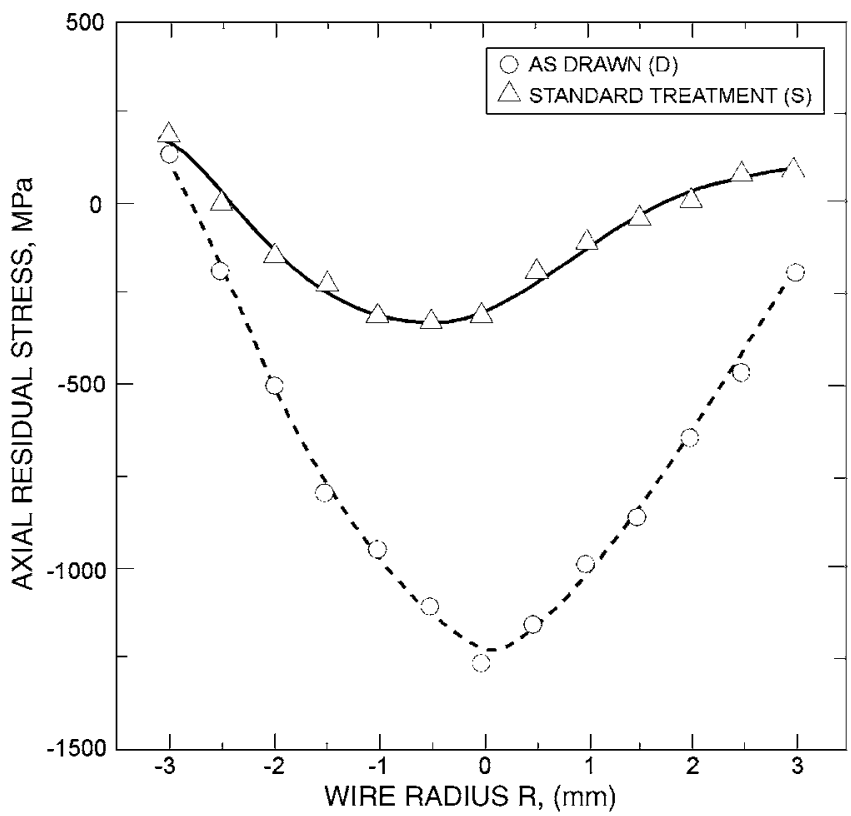

Fig. 10. Axial residual stress as a function of the radial coordinate. Comparison between the "as-drawn" (D) sample and the standard treatment (S) sample.

of residual stresses would be welcome for improving the final treatments.

The effect of the stretching force during the post-drawing thermomechanical treatment is shown in Fig. 11, where the standard treatment $(\mathrm{S})$ provides better results than the treatment with lower force (LF). However, it seems that the residual stresses can be further reduced by increasing the stretching force (HF), as shown in the corresponding profile, which is almost flat. However, this reduction scarcely affects the tensile properties, as can

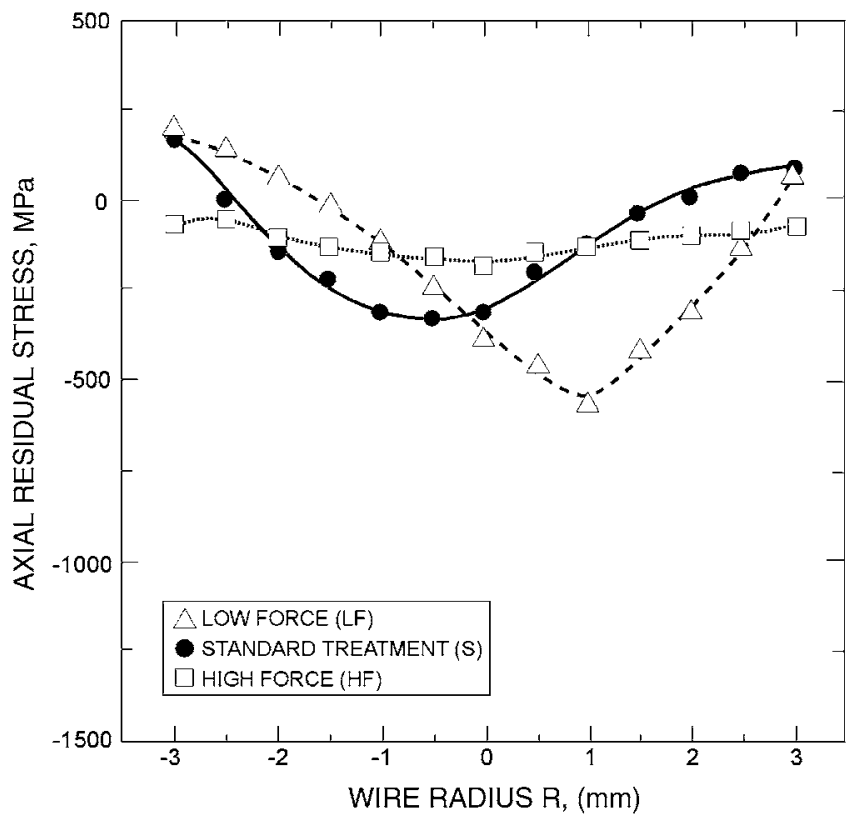

Fig. 11. Effect of the stretching force in the axial residual stress as a function of the radial coordinate. Comparison between the standard treatment $(\mathrm{S})$, low-force (LF) and high-force (HF) samples.

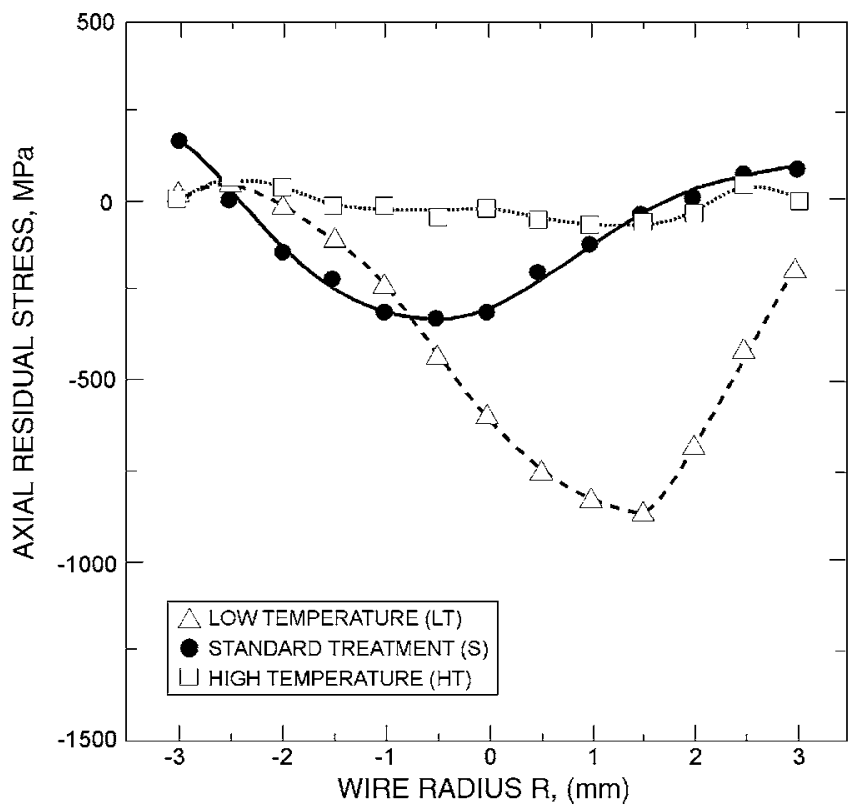

Fig. 12. Effect of the temperature in the axial residual stress as a function of the radial coordinate. Comparison between the standard treatment $(S)$, lowtemperature (LT) and high-temperature (HT) samples.

be noticed in Table 2 . The only remarkable variation is a small increment in the conventional yield stress $\sigma_{0.2}$.

The effect of the temperature during the post-drawing thermomechanical treatment is shown in Fig. 12, where the longitudinal stress profiles for the ferrite phase are compared. Clearly the low-temperature treatment (LT) produces poor results. It seems that the temperature is insufficient to noticeably reduce the stresses, nor the wire curvature (this may be the reason for the asymmetry of the profiles, as already mentioned). The standard treatment (S) appears satisfactory, however if the temperature is increased (HT) the results are better and the differences between stresses at the surface and at the centre become almost negligible. In this case, the variations of temperature induce changes in the tensile properties, as shown in Table 2. The low-temperature treatment (LT) increases yield stress and decreases ductility (measured as elongation under maximum load). On the other hand, at high temperature, ductility increases but yield and tensile stress decrease. It is worth remarking that although the $\mathrm{HT}$ treatment is very effective in reducing residual stresses, changes in yield $\left(\sigma_{0.2}\right)$ and tensile stresses $\left(\sigma_{\max }\right)$ provide values of $\sigma_{0.2} / \sigma_{\max }(0.839)$ unacceptable by standards that require $\sigma_{0.2} / \sigma_{\max }>0.85$

As previously mentioned, it has been unfeasible to measure the residual stresses in the cementite phase due to the poor peak statistics. Nevertheless, an average value of the axial stress in the cementite phase can be computed from the knowledge of the longitudinal values of the ferrite phase with some additional hypotheses.

The average value, in the cross-section, of the axial residual stress in the ferrite phase, $\sigma_{\mathrm{f}}^{\mathrm{m}}$, can be computed from

$2 \pi \int_{r=0}^{R} r \sigma_{\mathrm{f}} \mathrm{d} r=\pi R^{2} \sigma_{\mathrm{f}}^{\mathrm{m}}$ 
Table 3

Average values of axial residual stresses for ferrite and cementite

\begin{tabular}{lllllll}
\hline Batch & D & S & LF & HF & LT & HT \\
\hline$\sigma_{\mathrm{f}}^{\mathrm{m}}(\mathrm{MPa})$ & -400 & 30 & 20 & -60 & -150 & 50 \\
$\sigma_{\mathrm{c}}^{\mathrm{m}}(\mathrm{MPa})$ & 3600 & -270 & -180 & 660 & 1350 & -450 \\
\hline
\end{tabular}

where $\sigma_{\mathbf{f}}(r)$ is the value of the longitudinal stress at a distance $r$ from the centre of the section, and an axisymmetric stress distribution is assumed.

The average value of the residual stress in the cementite phase, $\sigma_{\mathrm{c}}^{\mathrm{m}}$, can be obtained from the force equilibrium requirement, the knowledge of the volumetric fraction of the ferrite, $f$ $(f=0.9$ for the steels used in this research), and assuming the rule of mixtures:

$(1-f) \sigma_{\mathrm{c}}^{\mathrm{m}}+f \sigma_{\mathrm{f}}^{\mathrm{m}}=0$

The computed average values of the ferrite and cementite phases for the as drawn and the five batches considered in this research are shown in Table 3. It can be seen that the average axial stresses in the cementite can be very high, reaching around $3600 \mathrm{MPa}$ in the "as-drawn" (D) sample. The mechanical behaviour of the cementite lamellae inside the pearlitic structure is not well known, although it seems to be very different from "bulk" cementite. The studies of Gil-Sevillano [21] show that the lamellar cementite should have some ductility to explain the deformation and the tensile strength reached by cold-drawn pearlite. In fact he concludes that the fine pearlitic structure and the important compression stress components allow the cementite to be ductile in cold-drawn pearlite. It has also been demonstrated the influence of the hydrostatic component of cold-drawing in the deformability and mechanical behaviour of cementite . Regarding the tensile strength of cementite in fine pearlite, there are a number of studies where cementite stresses similar or higher than $3600 \mathrm{MPa}$ have been reported

From Figs. 11 and 12 it appears that treatments at high force (HF) and at high temperature (HT) provide a good smoothing of the profile of residual stresses. Table 3 can supply additional information in relation with the mechanical behaviour during a tensile test. It was found that the yield stress for the HT batch was much lower than the values from other batches (see Table 2). This might be due to microstructural changes associated to the HT treatment, although residual stress might also play a role. In fact, the average value of the axial residual stress (in the soft ferrite phase) in the HT sample is $50 \mathrm{MPa}$ - the highest average tensile stress of all five batches. When an external tensile stress is applied, it adds to the residual stress until yielding is reached. Consequently, the higher the residual stress in the axial direction the sooner yielding will be reached.

It is apparent that the knowledge of residual stress profiles of both phases (ferrite and cementite) would be desirable to evaluate the effectiveness of the thermomechanical treatments. Nevertheless, if the residual stress cannot be measured in the cementite phase, as in our case, still some valuable results can be obtained from ferrite alone. From this point of view, neutron diffraction is a very powerful technique to improve thermome- chanical treatments based on quantitative data instead of the empirical procedures used nowadays.

\section{Conclusions}

The thermomechanical treatments are successful in relieving the residual stresses created by cold-drawing, although the role of the temperature and the stretching force are not completely understood. The standard treatment (S) is satisfactory. The treatments with less force (LF) or lower temperature (LT) are less reliable. If the temperature (HT), or especially the force (HF), is increased, the results are much better. It can be seen that the residual stresses in the ferrite are almost negligible in both cases. The best results are found when the force is increased (HF). In this case, all stress components are slightly compressive in the ferrite phase, with a minimum around $-200 \mathrm{MPa}$. This means that the cementite phase must be in tension to fulfill the load balance.

The technique based on neutron diffraction allows quantitative analysis of the residual stress profiles across the wire section. This procedure enables, in principle, the evaluation of the stresses in the different phases of the material and an assessment of the role of the temperature and stretching force during the thermomechanical treatments after cold-drawing. Consequently, this technique may be a useful tool to develop thermomechanical treatments based on quantitative measures instead of the empirical procedures used nowadays.

\section{Acknowledgements}

The Spanish Ministry of Education ("Ministerio de Educación, Cultura y Deporte") is gratefully acknowledged for supporting this research through the projects MAT2002-04343 and ENE2005-06478/CON. The authors are indebted to the EU for funding support to perform the neutron scattering measurements through the Neutron and Muon Integrated Infrastructure Initiative (NMI3). The authors are also very grateful for the help of Mr. Luis del Pozo (EMESA) for providing the material for this investigation and the mechanical properties of the different rods. The present work was conducted within the framework provided by the projects DUMEINPA, sponsored by the Comunidad de Madrid, Spain, and SEDUREC, integrated in the Spanish national research program CONSOLIDER-INGENIO 2010.

\section{References}

M. Zelin, Acta Mater. 50 (2002) 4431-4447.

J. Gil-Sevillano, J. Phys. III 1 (1991) 967-988.

M. Elices, J. Mater. Sci. 39 (2004) 3889-3899.

A.B. Dove, Wire J. Int. (1983) 58-65.

P. Renz, W. Steuff, R. Kopp, Wire J. Int. (1996) 64-69.

J.M. Atienza, M.L. Martinez-Perez, J. Ruiz-Hervias, F. Mompean, M. Garcia-Hernandez, M. Elices, Scripta Mater. 52 (2005) 305-309.

J.M. Atienza, J. Ruiz-Hervias, M.L. Martinez-Perez, F.J. Mompean, M. Garcia-Hernandez, M. Elices, Scripta Mater. 52 (2005) 1223-1228.

H. Godfrey, F. Richards, S. Sason, Wire J. Int. (2000) 102-113.

J.M. Atienza, M. Elices, Mater. Struct. 37 (2004) 301-304.

J. Llorca, V. Sanchez-Galvez, Fatigue Fract. Eng. M. 12 (1989) 31-45 
I. Verpoest, E. Aernoudt, A. Deruyttere, M. Debondt, Int. J. Fatigue 7 (1985) 199-214.

M. Elices, G. Maeder, V. Sanchez-Galvez, Br. Corros. J. 18 (1983) $80-81$.

M. Elices, J. Ruiz, J.M. Atienza, Mater. Struct. 37 (2004) 305-310.

J. Ruiz-Hervias, V. Luzin, H. Prask, T. Gnaeupel-Herold, M. Elices, Mater. Sci. Eng. A: Struct. 435/436 (2006) 725-735.

J. Ruiz, J.M. Atienza, M. Elices, J. Mater. Eng. Perform. 12 (2003)480-489. J.R. Santisteban, M.R. Daymond, J.A. James, L. Edwards, J. Appl. Crystallogr. 39 (2006) 812-825.

ENGIN-X Script Based Analysis-Open Genie based data analysis suite, http://www.isis.rl.ac.uk/engineering/download/download.htm.

I.C. Noyan, J.B. Cohen, Residual Stress: Measurement by Diffraction and Interpretation, Springer-Verlag New York Inc., Berlin, 1987.
T. Gnaupel-Herold, P.C. Brand, H.J. Prask, J. Appl. Crystallogr. 31 (1998) 929-935.

M.L. Martinez-Perez, C.R. Borlado, F.J. Mompean, M. Garcia-Hernandez, J. Gil-Sevillano, J. Ruiz-Hervias, J.M. Atienza, M. Elices, R.L. Peng, M.R. Daymond, Acta Mater. 53 (2005) 4415-4425.

J. Gil Sevillano, Mater. Sci. Eng. 21 (1975) 221-225.

G. Lanford, Metall. Trans. A 8A (1977) 861-875.

J. Gil Sevillano, P. Van Houtte, E. Aernoudt, Prog. Mater. Sci. 25 (1980) $69-412$.

Y. Tomota, P. Lukas, D. Neov, S. Harjo, Y.R. Abe, Acta Mater. 51 (2003) 805-817.

K. Van Acker, J. Root, P. Van Houtte, E. Aernoudt, Acta Mater. 44 (1996) 4039-4049.

M.R. Daymond, H.G. Priesmeyer, Acta Mater. 50 (2002) 1613-1626. 